\title{
Perspective \\ Co-Creation Facilitates Translational Research on Upper Limb Prosthetics
}

\author{
Hannah Jones ${ }^{1,2, *}$, Sigrid Dupan ${ }^{1}\left(\mathbb{D}\right.$, Maxford Coutinho ${ }^{3}$, Sarah Day ${ }^{4}$, Deirdre Desmond ${ }^{5}$, \\ Margaret Donovan-Hall ${ }^{6}$ D , Matthew Dyson ${ }^{2}\left(\mathbb{D}\right.$, Thea Ekins-Coward ${ }^{2}$, Laurence Kenney ${ }^{7}$ (D), \\ Agamemnon Krasoulis ${ }^{2}$, Doug McIntosh ${ }^{8,+}$, Kaveh Memarzadeh ${ }^{8,+} \mathbb{D}$, Edward Small ${ }^{9}$, Gemma Wheeler ${ }^{10} \mathbb{D}$, \\ Hancong $\mathrm{Wu}^{1}{ }^{1}$ a and Kianoush Nazarpour ${ }^{1, *(\mathbb{D})}$
}

check for

updates

Citation: Jones, H.; Dupan, S.; Coutinho, M.; Day, S.; Desmond, D.; Donovan-Hall, M.; Dyson, M.;

Ekins-Coward, T.; Kenney, L.; Krasoulis, A.; et al. Co-Creation Facilitates Translational Research on Upper Limb Prosthetics. Prosthesis 2021, 3, 110-118. https://doi.org/ $10.3390 /$ prosthesis3020012

Academic Editor: Winnie Jensen

Received: 4 February 2021

Accepted: 24 March 2021

Published: 2 April 2021

Publisher's Note: MDPI stays neutral with regard to jurisdictional claims in published maps and institutional affiliations.

Copyright: (C) 2021 by the authors Licensee MDPI, Basel, Switzerland. This article is an open access article distributed under the terms and conditions of the Creative Commons Attribution (CC BY) license (https:// creativecommons.org/licenses/by/ $4.0 /)$.
1 Edinburgh Neuroprosthetics Laboratory, School of Informatics, The University of Edinburgh, Edinburgh EH8 9YL, UK; sigrid.dupan@ed.ac.uk (S.D.); hancong.wu@ed.ac.uk (H.W.)

2 School of Engineering, Newcastle University, Newcastle upon Tyne NE1 7RU, UK; matthew.dyson@ncl.ac.uk (M.D.); thea.ekins-coward@hotmail.com (T.E.-C.); agamemnon.krasoulis@ncl.ac.uk (A.K.)

3 Department of Plastic Surgery, Royal Victoria Infirmary, Newcastle upon Tyne NE1 4LP, UK; mdrcoutinh@aol.com

4 Department of Biomedical Engineering, University of Strathclyde, Glasgow G1 1QE, UK; sarah.day@strath.ac.uk

5 Department of Psychology, Maynooth University, W23 F2K8 Maynooth, Ireland; Deirdre.Desmond@mu.ie

6 School of Health Sciences, University of Southampton, Highfield Southampton SO17 1BJ, UK; mh699@soton.ac.uk

7 School of Health and Society, University of Salford, Manchester M5 4WT, UK; L.P.J.Kenney@salford.ac.uk

8 Authors in a Personal Capacity, UK; doug.mcintosh@btinternet.com (D.M.); kmemarzadeh84@gmail.com (K.M.)

9 School of Humanities, University of Dundee, Dundee DD1 4HN, UK; e.z.small@dundee.ac.uk

10 National Institute of Health Research Children and Young People MedTech Co-operative, Sheffield S10 2TH, UK; gemma.wheeler@nihr.ac.uk

* Correspondence: hannah.jones@ed.ac.uk (H.J.); kianoush.nazarpour@ed.ac.uk (K.N.)

+ Authors in a personal capacity.

Abstract: People who either use an upper limb prosthesis and/or have used services provided by a prosthetic rehabilitation centre, hereafter called users, are yet to benefit from the fast-paced growth in academic knowledge within the field of upper limb prosthetics. Crucially over the past decade, research has acknowledged the limitations of conducting laboratory-based studies for clinical translation. This has led to an increase, albeit rather small, in trials that gather real-world user data. Multi-stakeholder collaboration is critical within such trials, especially between researchers, users, and clinicians, as well as policy makers, charity representatives, and industry specialists. This paper presents a co-creation model that enables researchers to collaborate with multiple stakeholders, including users, throughout the duration of a study. This approach can lead to a transition in defining the roles of stakeholders, such as users, from participants to co-researchers. This presents a scenario whereby the boundaries between research and participation become blurred and ethical considerations may become complex. However, the time and resources that are required to conduct co-creation within academia can lead to greater impact and benefit the people that the research aims to serve.

Keywords: co-creation; collaboration; upper limb prosthetics; user needs

\section{Introduction}

The aim of the paper is to present a perspective of how co-creation can enable collaboration with multiple stakeholders throughout a research study. Within the field of upper limb prosthetics, we share our perspective on how co-creation can facilitate a transition from short-term laboratory-based testing, to long-term in-home trials. Additionally, we 
offer our broad perspective on the challenges and opportunities of how co-creation can contribute to the translation of academic knowledge into clinical practice.

Translating research into clinical practice for the benefit of users of upper limb prosthetics has been notoriously slow. As aforementioned, with the term "users" we refer to people who either use an upper limb prosthesis and/or have used the services provided by a prosthetic rehabilitation centre. The last two decades have seen increased academic interest in prosthesis user needs and satisfaction [1-18]. However, proposed advancements that address the identified needs have not led to a reduction in device abandonment, which has highlighted the gap between published research and its translation into clinical practice $[6,19]$. Furthermore, evidence has highlighted that laboratory metrics and findings are not always consistent with clinical and/or outcomes of importance to users [20]. This exposes a limited understanding of how devices and/or systems fit within the daily lives and routines of users, for example, within a home environment where experimental constraints are relaxed [21-23]. The current rate of progress in addressing user needs poses an opportunity to explore how different research approaches can lead to advancement [24]. By incorporating user-centred information throughout all stages of research, new knowledge can be generated that may reduce the gap between academic developments and positive impacts on prosthesis users' experience in their everyday lives.

\section{Background}

In recent years, translational healthcare research, which aims to translate research into practice to reach patients [25], has experienced benefits of expanding the range of collaborative stakeholders [26,27]. Within pharmaceutical development, open innovation has enabled collaboration between researchers, industry agencies, users, and policy makers [26]. Open innovation provides a framework that enables research teams to conduct external collaborations by sharing information, resources, and intellectual property [28]. Within healthcare, collaboration with patients and oncology nurses has resulted in studies that identify improvements for cancer diagnosis services, such as mobile applications [29]. Within assistive technology, studies have presented how using techniques to engage a range of stakeholders can assist in creating solutions that integrate into people's lives, for example, rehabilitation advancement for people with multiple sclerosis [30], and technological innovation for people living with dementia [31]. Living laboratories that monitor everyday activities of people within real-life scenarios have enabled researchers to evaluate the usability of healthcare technologies and assess health outcomes [32]. Participatory research methods implemented within living laboratories have provided an opportunity for users to share feedback and suggestions for solution improvements [32]. A consistent factor within these examples is the involvement of patients or users, as collaborators within the research process, and how their involvement informs research outcomes. Co-creation Figure 1 can be an approach to facilitate such a form of collaboration, in particular between researchers, users, and clinicians.

Co-creation is an approach that emerged from participatory design practice, with a focus on generating ideas with users as partners rather than subjects/participants [33]. The past two decades have seen an increase in co-creation within many fields, which has led to variations of definition $[33,34]$. Figure 1 presents the definition of co-creation that the article will use, which seeks to assist in translating new knowledge into healthcare practice [35]. The definition presents four processes that facilitate collaboration with multiple stakeholders throughout a study, which can be applied to prosthetic research.

Collaboration with academic, clinical, and industrial stakeholders throughout a study can lead to academic publication and intellectual property in the short term. Broadening the range of stakeholders to include users, policy makers, and representatives from charitable and/or non-governmental organisations can facilitate the translation of knowledge into clinical practice in the long term [36]. This is especially evident when users directly contribute towards research studies by providing input based on their lived experiences [37]. Collaboration with users can lead to collecting real-world data, which can inform health- 
care policy evidence $[38,39]$. The implementation of co-creation throughout the duration of research studies can lead towards a significant step-change in how research practice develops within the field of upper limb prosthetics.

\section{Co-Creation}

"The generation of new knowledge that is derived from the application of rigorous research methods that are embedded into the delivery of a program or policy (by researchers and a range of actors including service providers, service users, community organisations and policymakers) through four collaborative processes:

1. generating an idea (co-ideation);

2. designing the program or policy and the research methods (co-design);

3. implementing the program or policy according to the agreed research methods (co-implementation),

4. the collection, analysis and interpretation of data (co-evaluation)."

Figure 1. Co-creation definition. Quotation from Pearce [35].

\section{Application to Upper Limb Prosthetic Research}

The co-creation approach presented in Figure 2 presents an example of how multiple stakeholders can collaborative flexibly throughout a prosthetic research study. The approach, based on Pearce [35], was developed to present an example of (1) how the four collaborative processes of co-creation could occur over the duration of a research study, and (2) how a combination of stakeholders could collaborate throughout the approach.

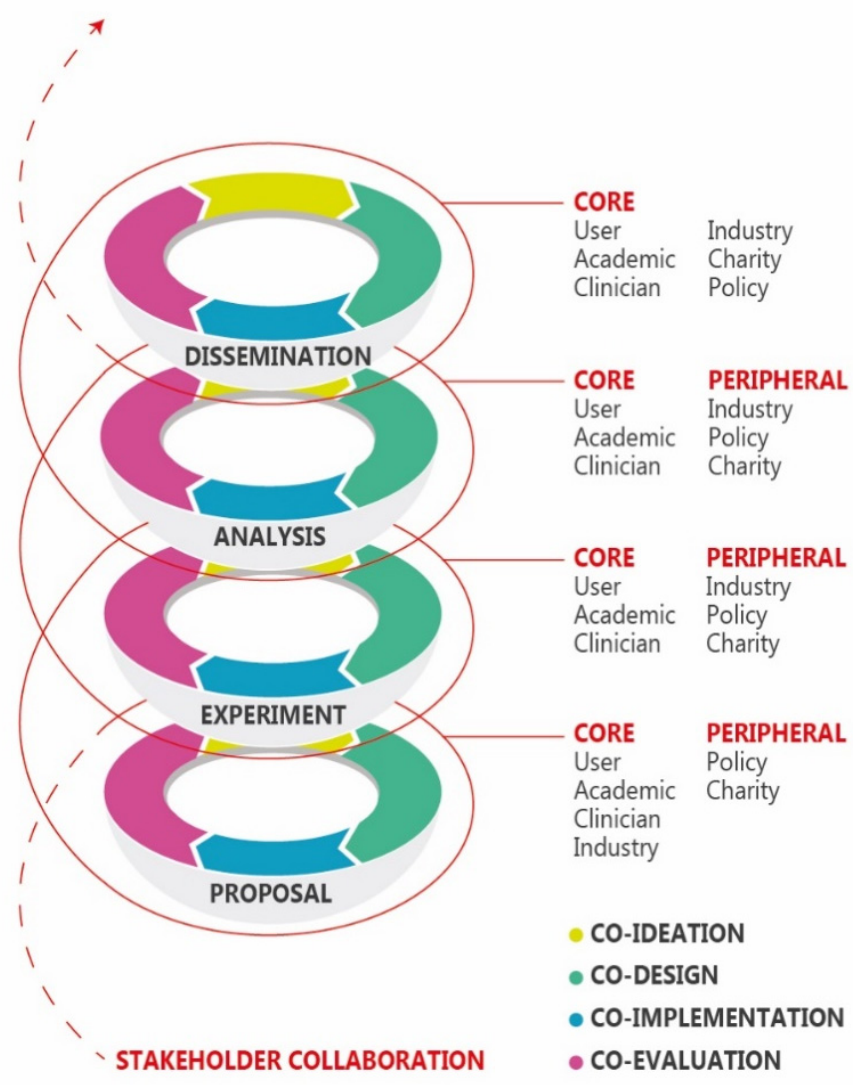

Figure 2. Co-creation model for upper limb prosthetics. 
All four collaborative processes of co-creation occur within each stage of prosthetic research, namely, research proposal development, experimental design, analysis of results, and dissemination of knowledge. In practice, research can be iterative, compared to the linear stages presented in Figure 2. However, Figure 2 presents an example of an adaptable reference point, based on the broad trajectory that research studies follow.

The core and peripheral stakeholders presented in Figure 2 document an example of how this approach could facilitate collaboration with a range of people throughout a study. The combination of stakeholders and their involvement in each collaborative process may vary at each research stage, depending upon the study, as illustrated in Figure 3. Core stakeholders are integral to the work within a study stage, by providing knowledge, expertise, and informed opinion. Collaboration with peripheral stakeholders occurs on an as-needed basis, with their participation contributing to the range of relevant opinions and expertise.

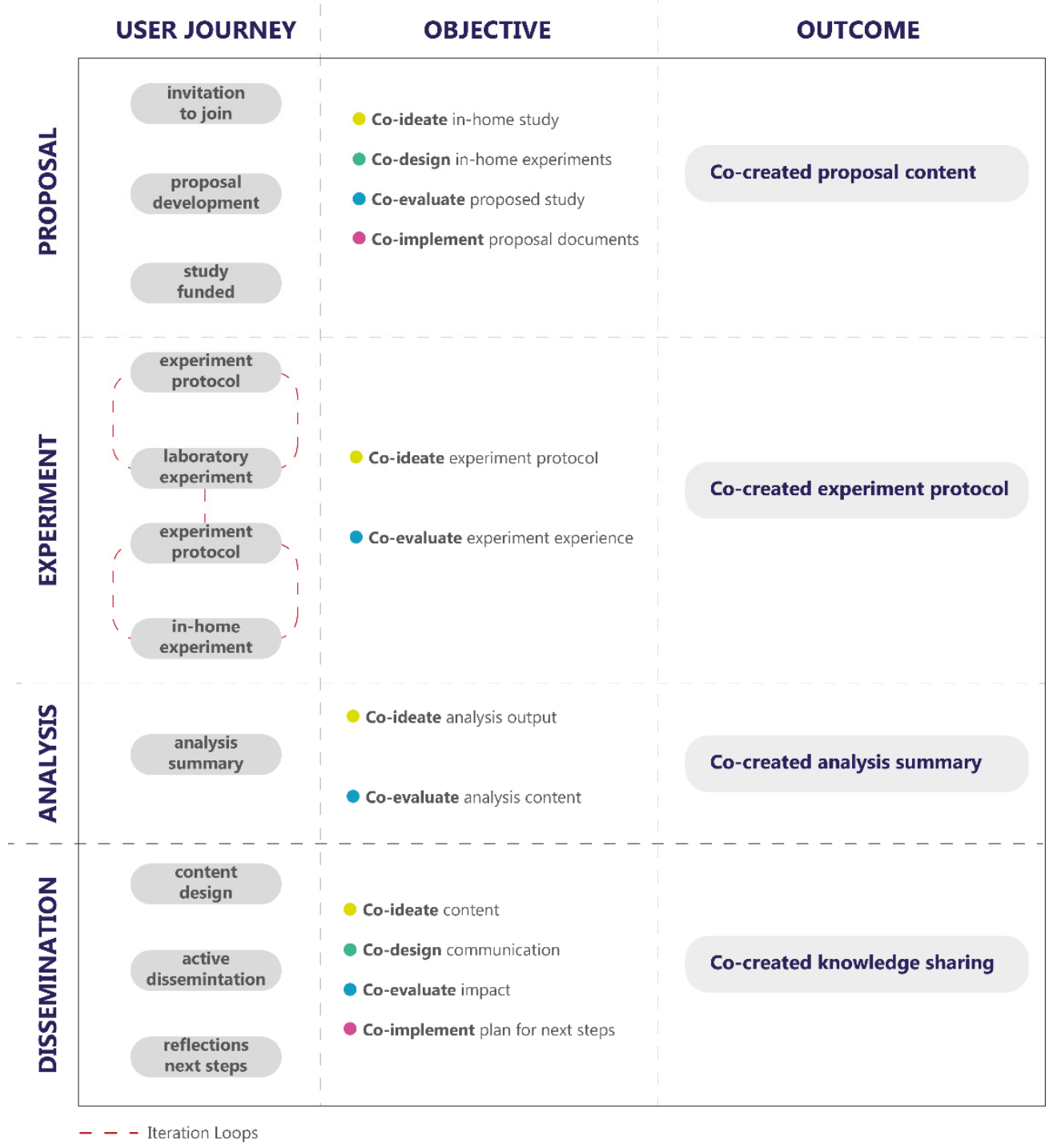

Figure 3. Co-creation for an in-home trial-a potential user journey map.

Based on the stakeholder combination presented in Figure 2, core stakeholders could become involved in the proposal stage by developing a collaborative proposed programme of research. Within the experiment stage, core stakeholders could collaborate to develop experimental protocols. Within the analysis stage, users could influence future studies by sharing their experience of the research, which could also inform published research within 
the dissemination stage. Figure 2 presents all stakeholders as core in the dissemination stage, as all parties can share research findings collaboratively and within their own respective fields. Furthermore, the involvement of policy makers at each stage of the approach enables research teams to create an on-going connection between research and policy. Figure 2, as aforementioned, is an example of a co-creation approach for upper limb prosthetics; other examples within the field are also documented, which present a range of collaborative stakeholders throughout a study [40].

An emerging route towards clinical translation for upper limb prosthetics is in-home trials [41], which presents a scenario whereby research transcends the laboratory, entering into home environments. Whilst in-home trials are technologically feasible [22,23,42,43], strategies that enable stakeholder collaboration within this context are not currently documented within the literature. Co-creation can be used as an approach to enable such collaboration; an example of which is presented in Figure 3, which illustrates one way that user involvement could occur throughout an in-home trial. The four stages of research presented in Figure 3 are in linear form; however, iteration across the stages and within the collaborative processes of co-creation is possible in practice. For example, during the proposal stage, users can influence the proposed research by providing feedback, and sharing experiences of using a prosthesis within the home. During the experiment stage, users can collaboratively develop experimental protocols, with a focus on the context of in-home trials, including opinions of remote data collection. The refinement of the protocol may require iteration, such as between co-ideate and co-evaluate within the experiment stage. Based on the experience of an in-home trial, users can influence the summary of findings within the analysis stage; including the identification of outcome measures in relation to the study. This can lead to forming recommendations for future studies. Furthermore, during the dissemination stage, users can contribute to peer reviewed articles and conference proceedings as co-authors and share research findings at outreach events.

Recruitment of users to in-home trials can be achieved through building relationships with existing research participants or forming new relationships through avenues, such as social media or connections to rehabilitation centres. Communication methods between researchers and users is key within the recruitment process. To that end, a variety of methods can be conducted, for example, project videos, text and pictorial documents, and phone and/or video conversations. Accessibility considerations are key within the recruitment process, ensuring that chosen mediums are accessible to all involved. Identifying clear inclusion criteria is beneficial when recruiting users to such studies. Given the longitudinal nature of in-home trials, which can run over several years, researchers and users can benefit from establishing how to work together and build relationships over a long-term basis. Initially, this can be addressed by defining stakeholder roles and responsibilities from the study onset.

Building collaborative relationships between researchers and users is based on forming trust through ensuring transparency between both parties and conducting ongoing informed consent [44]. Conducting research with users that incorporates user input from an early project stage can assist in forging strong relationships between stakeholders and lead to an effective research experience for all involved [40]. The key aspect of the model presented in Figure 2 is the identification of users as core stakeholders within all four study stages and the range of core stakeholders at the dissemination stage. This range of collaborative stakeholders can lead to greater success in translating academic research into clinical practice.

\section{Challenges and Opportunities}

The scenario presented above illustrates a transition in defining user involvement within research from participants to co-researchers. Such an approach comes with risks, for example, the blurring of the boundaries between research and everyday life could have a negative impact on users. Ethical considerations can therefore become more complex, for example, ensuring that meaningful, informed consent is obtained throughout the 
study [44]. In addition, the design of blinded experiments in collaboration with users is a pertinent consideration, such that biases are addressed. A key principle to be addressed and re-visited throughout the duration of a co-creation study is power sharing, which identifies research as jointly owned through neutral understanding [45]. Adopting an approach that involves users as core stakeholders will be challenging [37]. However, resources are available to assist the exploration of ethical considerations in collaboration with stakeholders throughout the course of a study, such as The Ethical Roadmap [46]. Furthermore, existing examples demonstrate that including users can lead to capturing new knowledge and experience that is vital in conducting meaningful, impactful research [47]. Therefore, it is timely to establish how co-creation can successfully work in practice.

\section{Suggestions for the Field}

Collaboration between academics and users is central to co-creation, which is supported by the definition of patient and public involvement: researching with people, rather than for people [48]. Conducting academic research with patients and/or the public has been facilitated by recent changes to ethical review mechanisms, which are proportionate to the level of risk within the study [49]. National and international research funders publish calls that require patient and public involvement within studies that are proposed. In addition, pairing schemes that provide opportunities to build knowledge of how policy is informed by research evidence can provide a platform to develop collaboration between academic and governmental partners [50]. However, a top-down approach can influence the challenges that are addressed by academic research, in addition to the research methods that are utilised. This is in contrast to a bottom-up structure whereby a population, such as a user group, defines a challenge and the methods that could be used to conduct research within that field. Consequently, a top-down approach can lead to tokenism, which may not result in co-created research that address the needs of a population [51]. Furthermore, the time required to implement co-creation can be deemed impractical, especially for early career researchers, due to contract duration and lack of career incentives for this area of research [52]. Given the multi-disciplinary nature of co-creation, from a broad perspective, academic institutions may benefit from assessing how studies are facilitated by current structures and career progression routes. Academic institutions can adopt a phased approach, which could be initiated by re-addressing incentives for conducting public involvement [52].

The rapid growth of online collaborative platforms during the Covid-19 pandemic has provided new possibilities to address the challenges of allocating time and identifying convenient locations to bring stakeholders together throughout a project. This is especially beneficial to users who require flexibility and balance in their own work and personal responsibilities, alongside their contribution to academic research. In addition, the technological capability of connecting multiple people through internet enabled devices, termed the Internet of Things, has provided remote methods of user interaction with clinical and academic teams within the field of rehabilitation [53]. Advancements in technology have enabled such forms of engagement within research; however, there are people who are digitally excluded from such studies, based on internet and device accessibility, and digital literacy [54].

Collaboration between academics and clinicians, as presented in Figure 2, can provide a bridge that connects researchers with users [55], which forms a collegiality whereby users can contribute throughout the research process.

\section{Conclusions}

The growth of user-centred studies within healthcare research generally presents an opportunity to identify approaches that can enable collaboration with multiple stakeholders throughout a study. Co-creation has the potential to aid in realising this opportunity, by identifying users as core stakeholders. Given the dynamic range of input that each stakeholder provides, the future of co-creation depends, in-part, on forming an inclusive 
approach that nurtures collaboration, especially between researchers and users. Now is the time to implement co-creation and frame its contribution towards enabling academic knowledge to translate into long-term user benefits and positive impacts, through the advancement of upper limb prosthetic devices and the overarching ecosystem of clinical care.

Author Contributions: All authors have contributed to the formation of the presented co-creation approach for translational research for upper limb prosthetics. Specifically, H.J., S.D. (Sigrid Dupan) and K.N. conceptualized the co-creation approach and the methodology outlined within the paper. T.E.-C., D.M. and E.S. contributed to the development of the presented approach, based on their experience of using an upper limb prosthetic device and receiving clinical care. H.J., S.D. (Sigrid Dupan) and K.N. created the visualization of the co-creation approach. HJ produced the original draft preparation. H.J., S.D. (Sigrid Dupan), K.N., D.D., M.D., G.W. and H.W. contributed to the writingreview and editing. All authors have read and agreed to the published version of the manuscript.

Funding: This work was supported in parts by the Mobility Matters initiative of the charity PORTER (Prosthetics, Orthotics and Rehabilitation Medicine-Education and Research) (Jones/2018), UK; Engineering and Physical Sciences Research Council (EPSRC), UK (EP/R004242/1); Newcastle University via the EPSRC Impact Acceleration Account (EP/R511584/1), UK; and Department of Health and Social Care (RNC/227/001), UK.

Acknowledgments: The authors would like to sincerely thank everyone who has participated in co-creation initiatives across all co-author affiliated institutions. The authors would also like to thank the Engineering and Physical Sciences Research Council (EPSRC), Department of Health and Social Care, and the PORT-ER charity for supporting this field of work.

Conflicts of Interest: The authors declare no conflict of interest.

Dedication: The authors wish to dedicate this paper in memory of Edward Small, who contributed to the original article, in shaping the idea of how co-creation can enable research to be conducted with users.

\section{References}

1. Nazarpour, K. Control of Prosthetic Hands: Challenges and Emerging Avenues, 1st ed.; IET Press: London, UK, 2020.

2. Jones, H.; Dupan, S.S.G.; Nazarpour, K. The Future of Prosthetics: A User Perspective. In Proceedings of the Trent International Prosthetics Symposium, Manchester, UK, 20-21 March 2019.

3. Engdahl, S.M.; Christie, B.P.; Kelly, B.M.; Davis, A.; Chestek, C.A.; Gates, D.H. Surveying the interest of individuals with upper limb loss in novel prosthetic control techniques. J. Neuroeng. Rehabil. 2015, 12, 1-11. [CrossRef]

4. Østlie, K.; Lesjø, I.M.; Franklin, R.J.; Garfelt, B.; Skjeldal, O.H.; Magnus, P. Prosthesis rejection in acquired major upper-limb amputees: A population-based survey. Disabil. Rehabil. Assist. Technol. 2011, 7, 294-303. [CrossRef] [PubMed]

5. Cordella, F.; Ciancio, A.L.; Sacchetti, R.; Davalli, A.; Cutti, A.G.; Guglielmelli, E.; Zollo, L. Literature Review on Needs of Upper Limb Prosthesis Users. Front. Neurosci. 2016, 10, 209. [CrossRef] [PubMed]

6. Kumar, D.K.; Jelfs, B.; Sui, X.; Arjunan, S.P. Prosthetic hand control: A multidisciplinary review to identify strengths, shortcomings, and the future. Biomed. Signal Process. Control. 2019, 53, 101588. [CrossRef]

7. Jang, C.H.; Yang, H.S.; Yang, H.E.; Lee, S.Y.; Kwon, J.W.; Yun, B.D.; Choi, J.Y.; Kim, S.N.; Jeong, H.W. A Survey on Activities of Daily Living and Occupations of Upper Extremity Amputees. Ann. Rehabil. Med. 2011, 35, 907-921. [CrossRef]

8. Kyberd, P.J.; Wartenberg, C.; Sandsjö, L.; Jönsson, S.; Gow, D.; Frid, J.; Almström, C.; Sperling, L. Survey of upper limb prosthesis users in Sweden and the United Kingdom. J. Prosthet. Orthot. 2007, 19, 55-62. [CrossRef]

9. Luchetti, M.; Cutti, A.G.; Verni, G.; Sacchetti, R.; Rossi, N. Impact of Michelangelo prosthetic hand: Findings from a crossover longitudinal study. J. Rehabil. Res. Dev. 2015, 52, 605-618. [CrossRef]

10. Engdahl, S.M.; Chestek, C.A.; Kelly, B.; Davis, A.; Gates, D.H. Factors associated with interest in novel interfaces for upper limb prosthesis control. PLoS ONE 2017, 12, e0182482. [CrossRef]

11. Sugawara, A.T.; Ramos, V.D.; Alfieri, F.M.; Battistella, L.R. Abandonment of assistive products: Assessing abandonment levels and factors that impact on it. Disabil. Rehabil. Assist. Technol. 2018, 13, 716-723. [CrossRef]

12. Biddiss, E.; Chau, T. Upper-Limb Prosthetics. Am. J. Phys. Med. Rehabil. 2007, 86, 977-987. [CrossRef]

13. Biddiss, E.A.; Chau, T.T. Upper limb prosthesis use and abandonment. Prosthet. Orthot. Int. 2007, 31, 236-257. [CrossRef] [PubMed]

14. Postema, K.; Van Der Donk, V.; Van Limbeek, J.; Rijken, R.A.; Poelma, M.J. Prosthesis rejection in children with a unilateral congenital arm defect. Clin. Rehabil. 1999, 13, 243-249. [CrossRef] [PubMed] 
15. Postema, S.G.; Bongers, R.M.; Brouwers, M.A.; Burger, H.; Hermansson, L.M.N.-; Reneman, M.F.; Dijkstra, P.U.; Van Der Sluis, C.K. Upper Limb Absence: Predictors of Work Participation and Work Productivity. Arch. Phys. Med. Rehabil. 2016, 97, 892-899. [CrossRef] [PubMed]

16. Davidson, J. A survey of the satisfaction of upper limb amputees with their prostheses, their lifestyles, and their abilities. J. Hand Ther. 2002, 15, 62-70. [CrossRef]

17. Chadwell, A.; Kenney, L.; Granat, M.H.; Thies, S.; Head, J.; Galpin, A.; Baker, R.; Kulkarni, J. Upper limb activity in myoelectric prosthesis users is biased towards the intact limb and appears unrelated to goal-directed task performance. Sci. Rep. 2018, 8, 11084. [CrossRef]

18. Biddiss, E.; Beaton, D.; Chau, T. Consumer design priorities for upper limb prosthetics. Disabil. Rehabil. Assist. Technol. 2007, 2, 346-357. [CrossRef]

19. Salminger, S.; Stino, H.; Pichler, L.H.; Gstoettner, C.; Sturma, A.; Mayer, J.A.; Szivak, M.; Aszmann, O.C. Current rates of prosthetic usage in upper-limb amputees-Have innovations had an impact on device acceptance? Disabil. Rehabil. 2020, 1-12. [CrossRef]

20. Vujaklija, I.; Roche, A.D.; Hasenoehrl, T.; Sturma, A.; Amsuess, S.; Farina, D.; Aszmann, O.C. Translating Research on Myoelectric Control into Clinics-Are the Performance Assessment Methods Adequate? Front. Neurorobotics 2017, 11, 7. [CrossRef]

21. Simon, A.M.; Turner, K.L.; Miller, L.A.; Hargrove, L.J.; Kuiken, T.A. Pattern recognition and direct control home use of a multi-articulating hand prosthesis. In Proceedings of the IEEE 16th International Conference on Rehabilitation Robotics (ICORR), Toronto, ON, Canada, 24-28 June 2019; pp. 386-391. [CrossRef]

22. Chadwell, A.; Diment, L.; Micó-Amigo, M.; Ramírez, D.Z.M.; Dickinson, A.; Granat, M.; Kenney, L.; Kheng, S.; Sobuh, M.; Ssekitoleko, R.; et al. Technology for monitoring everyday prosthesis use: A systematic review. J. Neuroeng. Rehabil. 2020, 17, 1-26. [CrossRef]

23. Wu, H.; Dyson, M.; Nazarpour, K. Arduino-Based Myoelectric Control: Towards Longitudinal Study of Prosthesis Use. Sensors 2021, 21, 763. [CrossRef]

24. Van Der Sluis, C.K.; Bongers, R.M. TIPS for Scaling up Research in Upper Limb Prosthetics. Prosthesis 2020, 2, 340-351. [CrossRef]

25. Woolf, S.H. The Meaning of Translational Research and Why It Matters. JAMA 2008, 299, 211-213. [CrossRef] [PubMed]

26. Seyhan, A.A. Lost in translation: The valley of death across preclinical and clinical divide-Identification of problems and overcoming obstacles. Transl. Med. Commun. 2019, 4, 1-19. [CrossRef]

27. Fernandez-Moure, J.S. Lost in Translation: The Gap in Scientific Advancements and Clinical Application. Front. Bioeng. Biotechnol. 2016, 4, 43. [CrossRef]

28. Chesbrough, H. The Future of Open Innovation. Res. Manag. 2017, 60, 35-38. [CrossRef]

29. Austin, J.; Drossaert, S.C.H.C.; van Dijk, J.; Mirkovic, J.; Børøsund, E.; Sanderman, R.; Schroevers, M.J.; Bohlmeijer, E.T. Cocreation phases in integrating top-down and bottom-up requirements: Developing a self-compassion app with cancer patients. In Supporting Health by Technology; University of Twente: Enschede, Netherlands, 2020; pp. 13-14.

30. Webster, A.; Poyade, M.; Rea, P.; Paul, L. The Co-design of Hand Rehabilitation Exercises for Multiple Sclerosis Using Hand Tracking System; Springer: Cham, Switzerland, 2019; Volume 1120, pp. 83-96.

31. Van den Kieboom, R.C.; Bongers, I.M.; Mark, R.E.; Snaphaan, L.J.; Mulvenna, M.; Kenning, G.; Nomali, M. User-Driven Living Lab for Assistive Technology to Support People With Dementia Living at Home: Protocol for Developing Co-Creation-Based Innovations. JMIR Res. Protoc. 2019, 8, e10952. [CrossRef]

32. Favela, J.; Kaye, J.; Skubic, M.; Rantz, M.; Tentori, M. Living Labs for Pervasive Healthcare Research. IEEE Pervasive Comput. 2015, 14, 86-89. [CrossRef]

33. Sanders, E.B.-N.; Stappers, P.J. Co-creation and the new landscapes of design. CoDesign 2008, 4, 5-18. [CrossRef]

34. De Koning, J.I.J.C.; Crul, M.R.M.; Wever, R. Models of co-creation. In Proceedings of the ServDes 2016, Copenhagen, Denmark, 24-26 May 2016; pp. 266-278.

35. Pearce, T.; Maple, M.; Shakeshaft, A.; Wayland, S.; McKay, K. What is the Co-Creation of New Knowledge? A Content Analysis and Proposed Definition for Health Interventions. Int. J. Environ. Res. Public Health 2020, 17, 2229. [CrossRef]

36. Monnard, K.; Benjamins, M.R.; Hirschtick, J.L.; Castro, M.; Roesch, P.T. Co-Creation of Knowledge: A Community-Based Approach to Multilevel Dissemination of Health Information. Health Promot. Pract. 2021, 22, 215-223. [CrossRef]

37. Van Der Scheer, L.; Garcia, E.; Van Der Laan, A.L.; Van Der Burg, S.; Boenink, M. The Benefits of Patient Involvement for Translational Research. Health Care Anal. 2014, 25, 225-241. [CrossRef] [PubMed]

38. Oliver, K.; Kothari, A.; Mays, N. The dark side of coproduction: Do the costs outweigh the benefits for health research? Health Res. Policy Syst. 2019, 17, 1-10. [CrossRef] [PubMed]

39. Berger, M.L.; Harnett, J. Are Real-World Data and Evidence Good Enough to Inform Health Care and Health Policy DecisionMaking? In Decision Making in a World of Comparative Effectiveness Research; Birnbaum, H., Greenberg, P., Eds.; ADIS: Singapore, 2017; pp. 97-103. [CrossRef]

40. Langley, J.; Wheeler, G.; Mills, N.; Heron, N. Starworks: Politics, power and expertise in co-producing a research, patient, practice and industry partnership for child prosthetics. In Proceedings of the Design4Health Conference, Amsterdam, The Netherlands, 1-3 July 2020; Volume 2, pp. 108-115.

41. Hargrove, L.J.; Miller, L.A.; Turner, K.; Kuiken, T.A. Myoelectric Pattern Recognition Outperforms Direct Control for Transhumeral Amputees with Targeted Muscle Reinnervation: A Randomized Clinical Trial. Sci. Rep. 2017, 7, 1-9. [CrossRef] [PubMed] 
42. Brinton, M.R.; Barcikowski, E.; Davis, T.; Paskett, M.; George, J.A.; Clark, G.A. Portable Take-Home System Enables Proportional Control and High-Resolution Data Logging With a Multi-Degree-of-Freedom Bionic Arm. Front. Robot. AI 2020, 7, 559034. [CrossRef]

43. Garske, C.A.; Dyson, M.; Dupan, S.; Nazarpour, K. Perception of Game-Based Rehabilitation in Upper Limb Prosthetic Training: Survey of Users and Researchers. JMIR Serious Games 2021, 9, e23710. [CrossRef]

44. Grant, A.D.; Wolf, G.I.; Nebeker, C. Approaches to governance of participant-led research: A qualitative case study. BMJ Open 2019, 9, e025633. [CrossRef]

45. Hickey, G.; Brearley, S.; Coldham, T.; Denegri, S.; Green, G.; Staniszewska, S.; Tembo, D.; Torok, K.; Turner, K. Guidance on Co-Producing a Research Project; NIHR INVOLVE: Southampton, UK, 2018.

46. The Ethical Roadmap. Available online: https:/ / ethicalroadmap.org/en/ (accessed on 25 February 2021).

47. Langley, J.; Wolstenholme, D.; Cooke, J. 'Collective making' as knowledge mobilisation: The contribution of participatory design in the co-creation of knowledge in healthcare. BMC Health Serv. Res. 2018, 18, 585. [CrossRef]

48. National Institute for Health Research. What is Public Involvement in Research? Available online: https://www.invo.org.uk/ find-out-more/what-is-public-involvement-in-research-2/ (accessed on 3 February 2021).

49. Jones, H.; Nazarpour, K. Gaining NHS ethical approval from the perspective of a biomedical engineering team. Br. J. Healthc. Manag. 2018, 24, 71-76. [CrossRef]

50. The Royal Society, Pairing Scheme. Available online: https://royalsociety.org/grants-schemes-awards/pairing-scheme/ (accessed on 3 February 2021).

51. Williams, O.; Sarre, S.; Papoulias, S.C.; Knowles, S.; Robert, G.; Beresford, P.; Rose, D.; Carr, S.; Kaur, M.; Palmer, V.J. Lost in the shadows: Reflections on the dark side of co-production. Health Res. Policy Syst. 2020, 18, 1-10. [CrossRef]

52. Burns, J.A.; Korzec, K.; Dorris, E.R. From intent to implementation: Factors affecting public involvement in life science research BioRxiv 2019, 748889. [CrossRef]

53. Gradim, L.C.; Jose, M.A.; Da Cruz, D.M.C.; Lopes, R.D.D. IoT services and applications in rehabilitation: An interdisciplinary and meta-analysis review. IEEE Trans. Neural Syst. Rehabil. Eng. 2020, 28, 1. [CrossRef]

54. Seah, K.M. COVID-19: Exposing digital poverty in a pandemic. Int. J. Surg. 2020, 79, 127-128. [CrossRef] [PubMed]

55. Fiorini, L.; Tabeau, K.; D'Onofrio, G.; Coviello, L.; De Mul, M.; Sancarlo, D.; Fabbricotti, I.; Cavallo, F. Co-creation of an assistive robot for independent living: Lessons learned on robot design. Int. J. Interact. Des. Manuf. 2019, 14, 491-502. [CrossRef] 